\section{FRI0745-HPR INVESTIGATION OF ASSOCIATION BETWEEN WRIST PAIN, FUNCTIONAL PERFORMANCE, GRIP AND PINCH STRENGTH IN CHILDREN AND ADOLESCENTS WITH JUVENILE IDIOPATHIC ARTHRITIS: CROSS-SECTIONAL STUDY}

S.N. Arman ${ }^{1}$, E. Tarakci ${ }^{2}$, O. Kasapcopur ${ }^{3} \cdot{ }^{1}$ Department of Physiotherapy and Rehabilitation, Faculty of Health Science; ${ }^{2}$ Department of Physiotherapy and Rehabilitation, Faculty of Health Science; ${ }^{3}$ Medical Faculty of Cerrahpasa, Department of Pediatric Rheumatology, Istanbul University, Istanbil, Turkey

Background: Juvenile idiopathic arthritis (JIA), among the most common chronic diseases of childhood, can be associated with pain. reduced range of motion, decreased muscle strength and functionality (1).

Objectives: The aim of this study was to assess the relationships between wrist pain and functional performance, grip and pinch strength in children and adolescents with JIA.

Methods: Cross-sectional study design was included 42 ( 36 female. 6 male) children and adolescents with JIA aged between 8 and 18 years. Patients who have at least one affected wrist included in the study. Sociodemographic data and clinical features were assessed by physical therapists. Hand grip strength was assessed with Jamar dynamometer Lateral, tip and palmar pinch strengths were assessed with Baseline pinchmeter. Wrist pain during the activity was evaluated with Numeric Rating Scale (NRS). A score of 0 indicated "no pain" and 10 indicated "extreme pain" for NRS. Functional performance of hand was assessed with "Jebson Taylor Hand Functional Test" (JTHFT). JTHFT consists 7 subtests: writing a 24-letter. card turning, picking up small common objects and placing them in a container, stacking checkers, stimulated feeding, moving light objects and moving heavy objects. All tests except writing performed on both right and left hand for JTHFT.

Results: The mean age and duration of disease was 13.05 \pm 3.04 (age range $8-18$ ) and $6.55 \pm 3.78$ years. respectively. Patient population consisted of 28 patients with polyarticular arthritis. 14 patients with oligoarticular arthritis. 39 of 42 patient's wrists were affected bilaterally. Significant relationships were found between writing and right palmar pinch strength $(r=-0.34 p=0.023)$, right hand grip strength and right stimulated feeding $(r=-0.32 \mathrm{p}=0.039)$, left hand grip strength and left moving heavy objects $(r=-0.33 p=0.028)$. Also, significant relationships were found between right lateral pinch strength and stimulated feeding $(r=-0.33$ $\mathrm{p}=0.028)$ and right stacking checkers $(\mathrm{r}=-0.31 \mathrm{p}=0.039)$. For right side, significant relationships were found between palmar pinch and moving light objects $(r=-0.35$ $p=0.020)$, moving heavy objects $(r=-0.32 p=0.039)$, stimulated feeding $(r=-0.36$ $\mathrm{p}=0.017)$, stacking checkers $(\mathrm{r}=-0.36 \mathrm{p}=0.017)$, card turning $(\mathrm{r}=-0.34 \mathrm{p}=0.026)$ and JTHFT-total $(r=-0.40 \mathrm{p}=0.008)$.

Conclusions: The results of our study showed that hand grip and pinch strengths considerably decreased in children and adolescents with JIA, according to the normal means of the grip strengths of healthy children and adolescents reported in the literature (2). Our study suggested that decreased hand grip and pinch strengths may result impairment daily functions but wrist pain does not effect grip strengths or functional performance in children and adolescents with JIA. Therefore, we conclude that the grip training program will be effective in improving the functional performance of children and adolescents with JIA.

References:

[1] Ravelli A, Martini A (2007) Juvenile idiopatic arthritis. Lancet 369 (9563):767778.

[2] Mathiowetz, Virgil, Diana M. Wiemer, and Susan M. Federman. Grip and pinch strength: norms for 6-to 19-year-olds. American Journal of Occupational Therapy 40.10 (1986): 705-711.

Disclosure of Interest: None declared

DOI: 10.1136/annrheumdis-2017-eular.4896

\section{FRI0746-HPR THE FACTORS AFFECTING STAIR CLIMBING IN PATIENTS WHO HAVE AN AFFECTED KNEE JOINT WITH OLIGOARTICULAR JUVENILE IDIOPATHIC ARTHRITIS}

S.N. Arman ${ }^{1}$, E. Tarakci ${ }^{1}$, O. Kasapcopur ${ }^{2} .{ }^{1}$ Division of Physiotherapy and Rehabilitation, Faculty of Health Science; ${ }^{2}$ Department of Pediatric Rheumatology, Medical Faculty of Cerrahpasa, Istanbul University, Istanbil, Turkey

Background: Juvenile idiopathic arthritis (JIA) encompasses a complex group of disorders with arthritis as a common feature. Patients with JIA may experience significant disability owing to muscular weakness, joint pain, contracture, and reduced mobility. Stair climbing performance is frequently used as a measure of lower extremity funtions in patients with chronic disease.

Objectives: The aim of this study was to investigating the factors affecting stair climbing in patients who have an affected knee joint with oligoarticular JIA.

Methods: 30 patients (aged 6-18 years) who have an affected knee joint with oligoarticular JIA were included in this study. Active range of motion (AROM) of knee was evaluated with universal goniometer. Muscular strength was estimated at maximal isometric force for the muscles of the lower extremities by using a portable handheld dynamometer (Lafayette, LA). Postural balance control was evaluated by using the Flamingo Balance Test (FBT). Static balance was measured by using the Functional Reach Test (FRT). Stair climbing performance was assessed with 10-stair climbing test (10SCT). All tests were performed thrice and the mean values of all were recorded. Relations between the factors affecting stair climbing in JIA were assessed by multiple linear regression analysis.

Results: The mean age was $9.63 \pm 2.76$ and the mean disease duration was $4.41 \pm 2.16$ years. Significant relationships were found between 10SCT and hip flexion muscle strength $(r=-0.45 p=0.013)$, hip external rotation muscle strength $(r=-0.48 \quad p=0.007)$, knee extension muscle strength $(r=-0.45 p=0.015)$ and dorsiflexion muscle strength $(\mathrm{r}=-0.37 \mathrm{p}=0.044)$. According to linear regression analysis, stair climbing performance was affected by only hip flexion muscle strength $(\beta=-0.40 \quad p=0.026)$. However, it was not found relationships between AROM, FRT, FBT and 10SCT ( $p>0.05)$.

Conclusions: Stair climbing is a biomechanically complex activity of daily life for children. In current study, it was found that stair climbing performance was related with lower extremity muscle strengths in patients with JIA. Althought patients with JIA had only one affected knee joint, hip flexion muscle strength was primary predictor of stair climbing performance. We suggested that stair climbing performance should be considered in patients with juvenile idiopathic arthritis. Thus, not only affected joint, but also all lower extremity joints should be assessed multidimensionally.

Disclosure of Interest: None declared

DOI: 10.1136/annrheumdis-2017-eular.4937

\section{FRI0747-HPR FEASIBILITY OF FOUR QUESTIONNAIRES TO EVALUATE PATIENT EDUCATION FOR PEOPLE WITH INFLAMMATORY ARTHRITIS}

$\underline{\text { R. Røe }}^{1}$, K. Gronning ${ }^{2}$, L.R. Eriksson ${ }^{1}$, H.A. Zangi ${ }^{3}$ on behalf of the Norwegian patient education expert panel. ${ }^{1}$ Dept. of Rheumatology, Diakonhjemmet Hospital, Oslo; ${ }^{2}$ Department of Public Health and Nursing, Center for Health Promotion Research, Norwegian University of Science and Technology, Trondheim; ${ }^{3}$ National Advisory Unit on Rehabilitation in Rheumatology, Dept. of Rheumatology, Diakonhjemmet Hospital, Oslo, Norway

Background: No core set of outcomes to evaluate patient education (PE) programmes exists. Evaluation of existing outcome measures is recommended ${ }^{1}$. A Norwegian expert panel comprising patients and health professionals experienced in patient education (PE) participated in a 3-round Delphi-process. The aim of the process was to obtain consensus about outcome domains that might be influenced by PE for people with inflammatory arthritis (IA). Six domains were identified: Understanding disease and treatment, knowledge about healthy life style, coping strategies, self-efficacy, empowerment and communication with health professionals. Through a systematic literature search and further Delphirounds, the expert panel identified four patient-reported outcome measures (PROMs) that might capture these outcome domains.

Objectives: To test the feasibility of four identified PROMs suitable to evaluate patient education programmes.

Methods: Arthritis Self-Efficacy Scales (ASES) for pain and symptoms, Effective Consumer Scale (EC-17), Health Education Questionnaire (heiQ) and Patient Activation Measure (PAM) were tested in 13 PE programmes for people with IA at six rheumatology departments in Norway. The PROMs were divided into two test-sets, set A (ASES, EC-17 and PAM) and set B (ASES and heiQ). Data were collected before, immediately after and 3 months after PE programmes. The instruments were tested for missing values, internal consistency (Cronbach's $\alpha)$ and ability to detect change. Floor and ceiling effects were considered to be present if more than $15 \%$ of the patients achieved the lowest or highest possible score on each item.

Results: 104 patients answered the questionnaires before participation in a PE programme, 63 in group A and 41 in group B, respectively. $96(92 \%)$ answered the questionnaires after intervention and $78(75 \%)$ responded at 3-month follow-up. Missing values were few in all PROMs (range 0-1.7\%). Cronbach's $\alpha$ was acceptable in ASES pain (0.75) and ASES symptoms (0.81), EC-17 (0.91) and PAM (0.78). HeiQ comprises eight separate subcategories: seven categories had acceptable Cronbach's $\alpha(0.72-0.86)$. All PROMs showed statistically significant improvements after the PE programmes. At 3-month followup statistically significant changes were found only in EC-17 ( $p=0.01$, SRM 0.4) and three heiQ subcategories; emotional distress ( $p=0.01$, SRM 0.5 ), skill and technique acquisition ( $p=0.02$, SRM 0.5$)$ and health service navigation $(p=0.02$, SRM 0.5). There were ceiling effects in all PROMs at baseline, 10/13 items in PAM, 5/17 in EC-17, 3/11 in ASES and 14/40 in heiQ. No floor effects were detected in any of the PROMs.

Conclusions: Based on this pilot study in patients with IA, EC-17 and three of the heiQ subcategories were the most feasible PROMs in terms of internal consistency and ability to detect change. Although ASES did not exhibit significant changes at 3-month follow-up, it should be considered as an outcome measure, because it is the only instrument to measure self-efficacy.

References:

[1] Zangi, H.A. et.al. EULAR recommendations for patient education for people with inflammatory arthritis. Ann Rheum Dis 2015;74:954-962.

Disclosure of Interest: None declared

DOI: 10.1136/annrheumdis-2017-eular.1706 